\title{
Simultaneous versus staged bilateral knee arthroplasty: do we impact chronic opioid use?
}

\author{
Alaa Abd-Elsayed
}

Wilson et $a l^{1}$ conducted a retrospective cohort study that included patients who underwent bilateral total knee arthroplasty (BTKA). Then they compared the postoperative chronic opioid use following simultaneous versus staged BTKA. Investigators found no statistically significant difference between both groups in postoperative chronic opioid use.

Anesthesiology Department, University of Wisconsin Madison School of Medicine and Public Health, Madison, Wisconsin, USA

Correspondence to Dr Alaa Abd-Elsayed, University of Wisconsin Madison School of Medicine and Public Health, Madison, Wisconsin, USA;

alaaawny@hotmail.com

Contributors AA-E is the sole author and contributed to the design and writing of this work.

Funding The author has not declared a specific grant for this research from any funding agency in the public, commercial or not-for-profit sectors.

\section{Competing interests None declared.}

Patient consent for publication Not required.

(c) American Society of Regional Anesthesia \& Pain Medicine 2021. No commercial re-use. See rights and permissions. Published by BMJ.

\section{A) Check for updates}

To cite Abd-Elsayed A. Reg Anesth Pain Med 2021;46:404.

\section{Received 13 November 2020}

Accepted 16 November 2020

Published Online First 6 January 2021

\section{(5) Linked}

- https://doi.org/10.1136/rapm-2020-102060

Reg Anesth Pain Med 2021;46:404.

doi:10.1136/rapm-2020-102294

\section{REFERENCE}

1 Wilson L, Fiasconaro M, Liu J, et al. Risk of chronic opioid use after simultaneous versus staged bilateral knee arthroplasty. Reg Anesth Pain Med 2021;46:405-9.

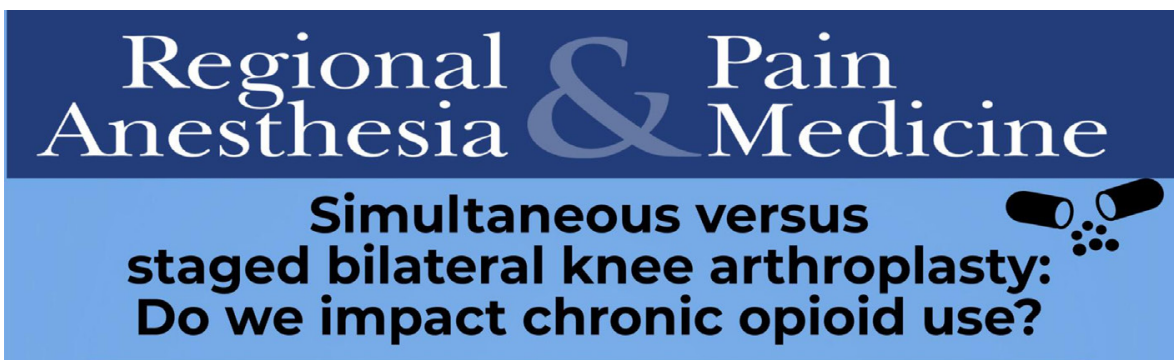

Wilson et al. evaluated the incidence of postoperative chronic opioid use following simultaneous versus staged BTKA, based on the different timing strategies of staged.

- This retrospective cohort study included patients who underwent bilateral total knee arthroplasty (BTKA) procedures $(n=14,407)$

- Cases were classified as having undergone simultaneous or staged BTKA ( $<3$ months, 3-6 months, or 6-12 months apart)

- Outcomes included postoperative chronic opioid use and oral morphine

\section{Conclusion}

Although patients undergoing staged BTKA $<6$ months apart were prescribed greater quantities of opioids upon discharge, there was no significant difference in the odds of postoperative chronic opioid use compared to simultaneous BTKA.

\section{Featured article:}

Wilson et.al. The risk of chronic opioid use after simultaneous versus staged bilateral knee arthroplasty dx.doi.org/10.1136/rapm-2020-102060

Infographic prepared by:

Alaa Abd-Elsayed, MD, MPH, FASA
Results

Unadjusted frequency of chronic opioid use did not differ between groups (Simultaneous: $11.3 \%$, Staged <3 months $10.7 \%$, Staged 3-6 months: $11.7 \%$, Staged $>6$ months: $10.2 \%$; $p=0.247$ ).
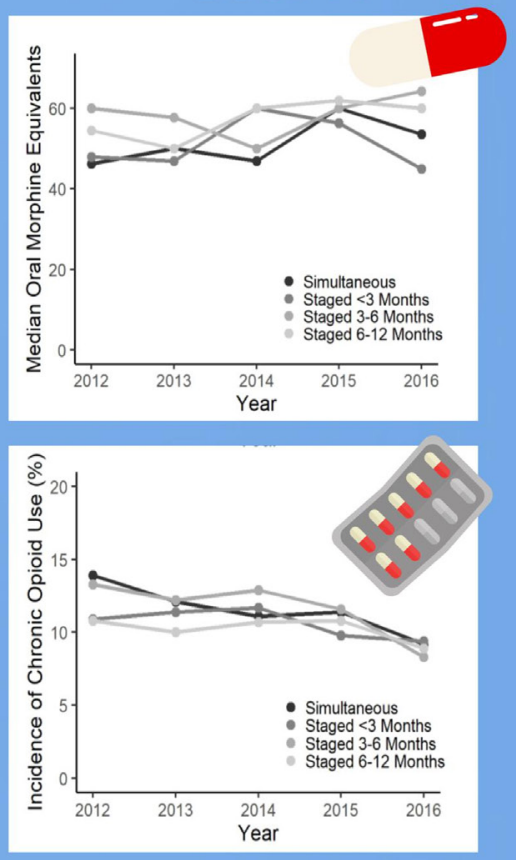